Liu, Suxing

\section{Differential expression of genes in human normal and breast cancer cells determined by microarray technique}

\author{
Suxing Liư ${ }^{1}, \mathbf{Q}$ un $\mathbf{W u}^{1}$, Paul Kirschmeier ${ }^{1}$, Terri McClanahan ${ }^{3}$, \\ Johnathan Greene ${ }^{2}$, Marco Hernandez ${ }^{2} \&$ Luquan Wang ${ }^{2}$ \\ ${ }^{1}$ Tumor Biology Department and ${ }^{2}$ Bioinformatics Group, Schering-Plough \\ Research Institute, 2015 Galloping Hill Road, \\ Kenilworth, New Jersey 07033 , USA \\ ${ }^{3}$ DNAX Research Institute, 901 California Avenue, \\ Palo Alto, California 94304, USA
}

The goal of this work is to study differential gene expression between normal breast epithelial cells and the malignant counterpart using microarray technique. We used GeneAlbum GEM 1 from Incyte Pharmaceuticals, Inc., which contains approximately 10,000 cDNA clones. 4 normal human mammary epithelial cells (HMEC) were used to search different gene expression patterns. In addition, 7 well-established breast cancer cell lines were used as probes against a common normal HMC.

Comparison between normal HMEC and malignant HMEC verified several known HMEC molecular markers, laminin-5 beta 3 and alpha 3, cytokeratins 5 , 15 and 17. We also observed some known breast cancer related genes, such as, HER2, HER3, and pS2, etc., were expressed as expected. The differential expression patterns among 4 normal HMEC, which may not be correlated with malignant phenotype, were used to reduce the background, or frequency of false "positive". In addition, microarray data analyses revealed that novel involvement of genes that are commonly differentially regulated in these breast cancer cells, such as, integrins alpha 3 and 6 .

\section{Livesey, Frederick}

\section{Clustering cell types and identifying cell- type specific transcripts by gene expres- sion profiling in single neurons}

\author{
F.J. Livesey ${ }^{1,2}$, S. Gustincich ${ }^{3}$, M. Steffen ${ }^{1}$, V. Cheung ${ }^{4}$, E. Raviola ${ }^{3}$, \\ G.M. Church ${ }^{1} \&$ C.L. Cepko ${ }^{1,2}$ \\ ${ }^{1}$ Department of Genetics, ${ }^{2}$ How ard Hughes M edical Institute and ${ }^{3}$ Department \\ of Neurobiology, Harvard M edical School, Boston, M assachusetts, USA \\ ${ }^{4}$ Department of Pediatrics, University of Pennsylvania, \\ Philadelphia, Pennsylvania, USA
}

At least one-third of the 100,000 genes in the mammalian genome are expressed only in the nervous system, but the diversity of types of neurons and the functional significance of this diversity is unknown. Given that much of what we know of the cell and molecular biology of neurons has been learned at the level of hundreds of thousands of cells, one essential tool for dissecting the diversity of neuronal types, and for understanding their function, is that of gene-expression profiling at the single-cell level. Therefore, we have used PCR-based methods for amplifying picogram quantities of complex RNA populations to synthesize and amplify cDNA from single, defined neurons from the mouse retina. To analyse these cDNA populations, we have used 1,100-element microarrays containing 130 known genes and 960 random clones from an adult mouse retina cDNA library. To test the ability of this system to distinguish between cell types and identify cellspecific genes, we are carrying out two types of experiments. To fingerprint cell types, we are comparing cDNA from each cell to a single reference sample of total brain cDNA and clustering cell types based on gene expression relative to entire brain. To directly isolate cell-type specific genes, we are also carrying out pair- wise comparisons of gene expression between each of the cell types and identifying genes whose expression is restricted to each cell type. Our studies demonstrate the feasibility of carrying out gene expression profiling experiments at the level of single cells in the nervous system and that such methods can be used to distinguish cell types.

Loftus, Stacie

\section{Informatic selection of a neural crest- melanocyte cDNA set for microarray analysis}

\section{S. Loftus ${ }^{1}$, Y. Chen², G. Gooden², J. Ryan ${ }^{3}$, G. Birznieks', M. Hilliard'1, A. Baxevanis ${ }^{3}$, M. Bittner', P. M eltzer',}

J. Trent ${ }^{2} \&$ W. Pavan ${ }^{1}$

${ }^{1}$ Genetic Disease Research Branch

${ }^{2}$ Cancer Genetic Branch

${ }^{3}$ Genome Technology Branch, National Institutes of Health, National Human Genome Research Institute, Bethesda, M aryland 20892, USA

While several genes, including three transcription factors (PAX3, MITF \& SOX10), that are essential for proper neural crest-melanocyte development in mouse and man have been identified, little is known about target gene regulation. Using cDNA microarrays it is now possible to the compare the expression of many genes simultaneously. We have taken advantage of the extensive sequence information in the dbEST to identify a neural crest-derived melanocyte (NC-M) cDNA set for microarray analysis. Analysis of characterised genes using dbEST identified one library (library 198) that contained ESTs representing 21 neural crest expressed genes and further analysis indicated the distribution of these genes was biased towards being derived from library 198. From library 198, we selected a subset of 852 clustered ESTs that have a library distribution profile similar to that of the 21 neural crest expressed genes. Microarray analysis demonstrated the majority of the neural crest-selected 852 ESTs were differentially expressed in melanoma cell lines compared to a non-neural crest kidney epithelial cell line ( $\mathrm{P}$ $\left.<1 \mathrm{X} 10^{-8}\right)$. This was not observed with an array of 1,238 ESTs that was selected without library origin bias $(\mathrm{P}=0.204)$. This study presents an approach for selecting tissue appropriate cDNAs that can be used to examine the expression profiles of developmental processes and diseases and for dissection of pathways involved in neural crest differentiation.

Loring, Jeanne

\section{Microarray analysis of gene expression pattems during neuronal differentiation of embryonic stem cells}

\author{
J.F. Loring ${ }^{1}$, R. Wesselschmidt' ${ }^{2}$ S. Fuhrman ${ }^{1}$, X. Wen ${ }^{1}$ \\ \& R. Somogyi ${ }^{1}$
}

${ }^{1}$ Neurobiology Dept., Incyte Pharmaceuticals, Inc., Palo Alto, California 94304, USA

${ }^{2}$ Transgenic Unit, Genome Systems, Inc., St. Louis, M issouri 63134, USA

The totipotence of embryonic stem (ES) cells makes them ideal for analysis of genes expressed during cellular differentiation. Some ES cell lines differentiate almost entirely to neurons in response to retinoic acid. We are studying changes in gene expression that accompany neuronal differentiation, using cDNA microarrays to compare the expression levels of nearly 9,000 mouse genes in undifferentiated and differentiated ES cells. We have defined a "gene expression signature" of ES-derived neurons, which includes approximately 100 genes that are up-reg- 